\title{
Visual Allesthesia in a Patient with Glioblastoma Multiforme
}

\author{
Antonios Reptsis ${ }^{1}$, Iordanis Demirtzoglou ${ }^{1 *}$, Athanasios Nikolakopoulos ${ }^{1}$, Diamantis Almaliotis ${ }^{2}$, \\ Angeliki Cheva, Vasileios Karampatakis ${ }^{2}$ \\ ${ }^{1}$ Eye Clinic, Papanikolaou General Hospital, Thessaloniki, Greece; ${ }^{2}$ Laboratory of Experimental Ophthalmology, Aristotle University \\ of Thessaloniki, Thessaloniki, Greece. \\ Email: *iordanisdemirtzoglou@yahoo.com
}

Received March 10 ${ }^{\text {th }}, 2012$; revised April 24 $4^{\text {th }}, 2012$; accepted May $8^{\text {th }}, 2012$

\begin{abstract}
Purpose: To report a rare case of visual allesthesia in a patient with glioblastoma multiforme. Material-Methods: A 46-year-old male presented in emergency ophthalmologic department complaining for difficulties in performing tasks related to color discrimination in his occupation (PC technician). The patient underwent a thorough ophthalmological examination and then he was referred to the neurological department for further evaluation. Results: The patient presented an atypical pattern of color perception disturbance. His best corrected visual acuity decreased progressively during hospitalization. He also experienced visual allesthesia paroxysmally (illusory left homonymous transpositions of subjects viewed in the right homonymous visual field). The visual field evaluation revealed homonymous left hemianopsia. Magnetic resonance imaging revealed glioblastoma multiforme confirmed by biopsy. Conclusion: A thorough ophthalmological and neuro-imaging control is suggested in patients with sudden color perception disturbance. Patients with temporal or occipital cortex damage may experience visual allesthesia.
\end{abstract}

Keywords: Glioblastoma Multiforme; Visual Allesthesia; Visual Field Defect

\section{Introduction}

Glioblastoma multiforme (GBM) is the most common and most malignant of the primary brain tumors. Several studies $[1,2]$ assess GBM prognosis associated with different mutations. GBM occurs most often in subcortical white matter of the temporal (31\%), parietal (24\%), frontal (23\%), and occipital (16\%) lobes [3]. Combined frontotemporal location is particularly typical. Brainstem, cerebellum, and spinal cord are less common sites for GBM. The etiology of GBM remains unknown. Familial gliomas account for approximately $5 \%$ of malignant gliomas and less than $1 \%$ of gliomas are associated with genetic disorders such as tuberous sclerosis, neurofibromatosis type 1 and type 2, Turcot syndrome, Li-Fraumeni syndrome [4].

Patients with no treatment die usually within 3 months. Patients with combination of surgical resection, radiotherapy and chemotherapy present a median survival of approximately 12 months, with fewer than $25 \%$ of patients surviving up to 2 years and fewer than $10 \%$ of patients surviving up to 5 years. While GBM occurs in all age groups, its peak incidence is at $45-70$ years with a mean age of 53 years [2]. Neurologic symptoms and

${ }^{*}$ Corresponding author. signs reflect the location, size and rate of tumor growth, including non specific headaches, nausea and vomiting, personality changes, cognitive impairment, hemiparesis, sensory loss, visual loss (visual field defects, as cortically based hemianopsia, may present in occipital lobe tumor location), aphasia and seizures.

We report a case of acquired dyschromatopsy as the initiative symptom and visual allesthesia in a patient with GBM.

\section{Case Report}

A 46-year-old male was referred by a private practice physician to the emergency department of our clinic. During a schedule color graphics installation on PC, he replaced 4 schedules considering them defective unless a technician colleague informed him that schedules were not defective. Color perception disturbance was the initial symptom in the presented patient with GBM and the patient revealed the experience of visual allesthesia a few days after hospitalization.

Patient did not use medications except for arterial hypertension and no history of familial tumors. Best corrected visual acuity was $10 / 10(-1.00 \mathrm{sph},-1.50 \mathrm{cyl} / 10$ degrees) for right eye and 10/10 (-1.25 sph, $-2.50 \mathrm{cyl} / 20$ 
degrees) for left eye. Intraocular pressure measured with Goldmann applanation tonometer was 14 and $15 \mathrm{mmHg}$ for right and left eye respectively. Slit lamp fundus examination and color fundus images obtained by fundus camera, revealed no abnormalities on optic nerve head appearance. There were no pupillary reflex and ocular movement abnormalities. Color blindness Ishihara test (Figure 1) and Fransworth-Munsell D-15 Hue test (Figure 2) confirmed color perception disturbance, however did not reveal a typical pattern of deficiency. Visual field evaluation by standard automated perimetry revealed homonymous left hemianopsia (Figure 3). Brain-CT (Figure 4) revealed a mass (glioma) located inside the right temporal-occipital lobe with peripheral zone enhancement and central necrosis, and mild compressive effects on the ventricular system without midline shift. MRI (Figure 5) revealed a $3 \times 6 \mathrm{~cm}$ mass (GBM) located inside the occipital lobe with central hypodense core, contrast enhancing ring and peripheral zone of non enhancing low attenuation. Definitive diagnosis of GBM was confirmed by biopsy (Figure 6). Patient was referred to neurologic and then to neurosurgery management.

\begin{tabular}{|c|c|c|c|c|c|}
\hline \multicolumn{3}{|c|}{ Right eye } & \multicolumn{3}{|c|}{ Left eye } \\
\hline Normal & Patient & Plates (pages) & Normal & Patient & Plates (pages) \\
\hline 57 & 15 & 5 & 57 & 67 & 5 \\
\hline 74 & 21 & 9 & 73 & 13 & 17 \\
\hline 97 & 7 & 12 & & & \\
\hline 45 & 15 & 13 & & & \\
\hline 73 & 13 & 17 & & & \\
\hline
\end{tabular}

Figure 1. Color blindness Ishihara test (38 plates edition) on right and left eye indicating atypical color perception disturbance.

\section{TEST 15 HUE DESATURE de LANTHONY selon FARNSWORTH-MUNSELL}

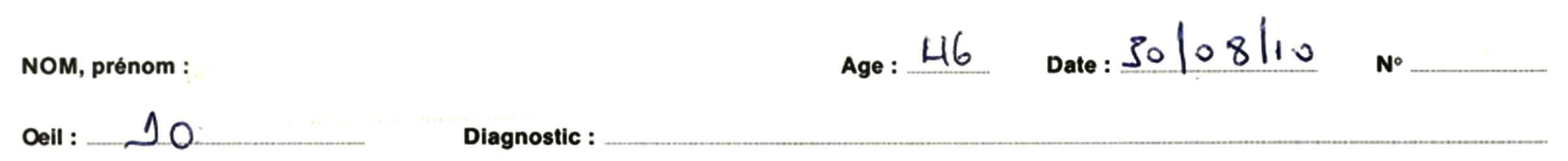

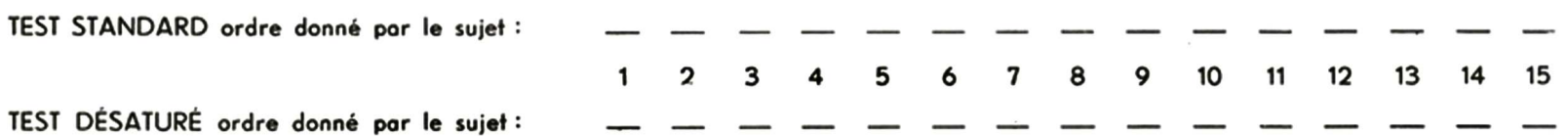

TEST STANDARD

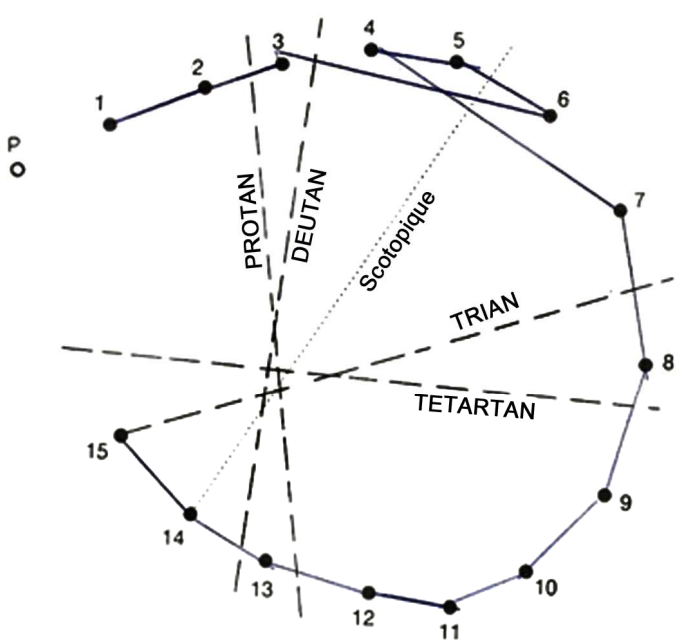

TEST DESATURE

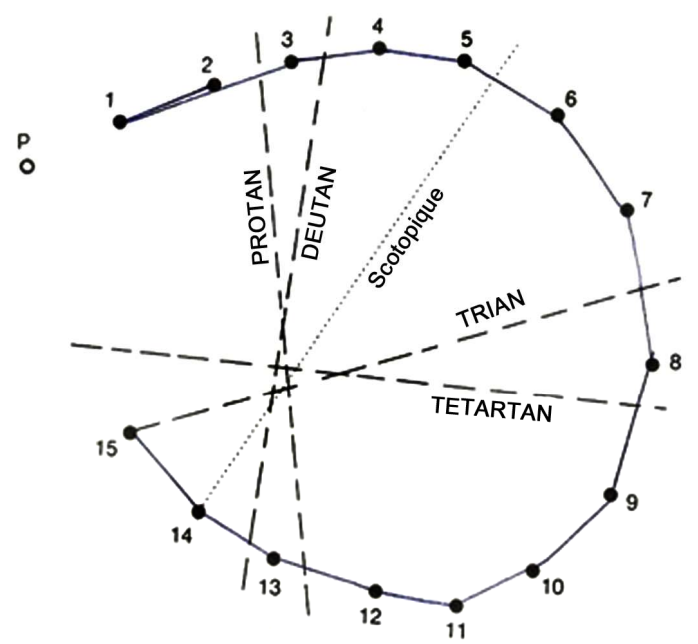

(a) 


\section{TEST 15 HUE DESATURE de LANTHONY selon FARNSWORTH-MUNSELL}
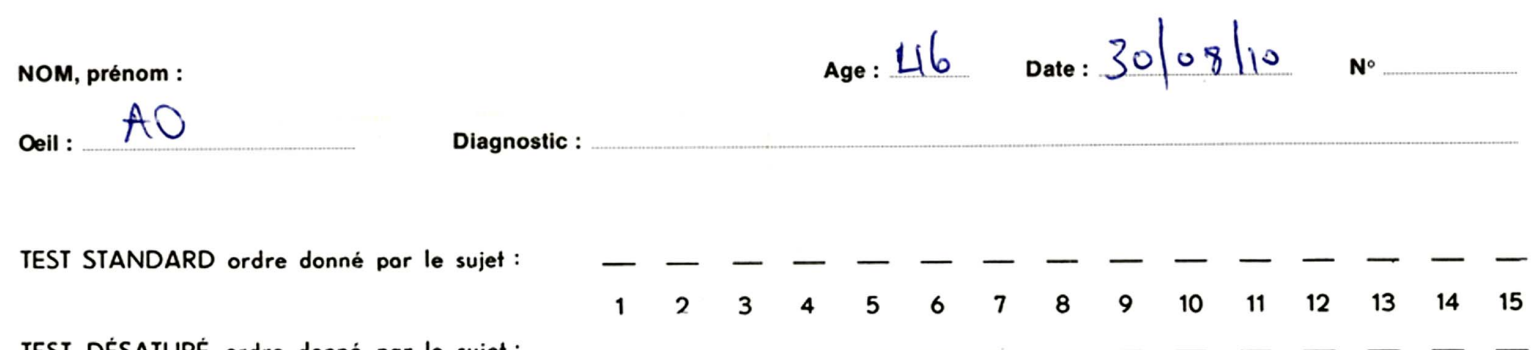

TEST STANDARD

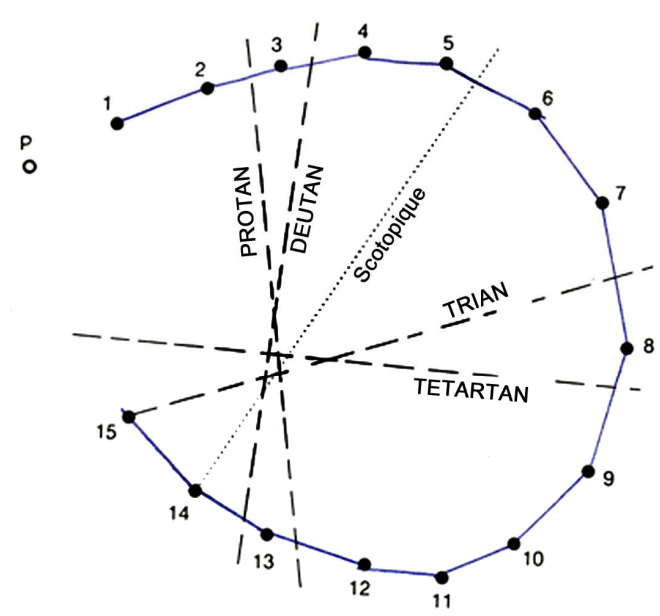

TEST DESATURE

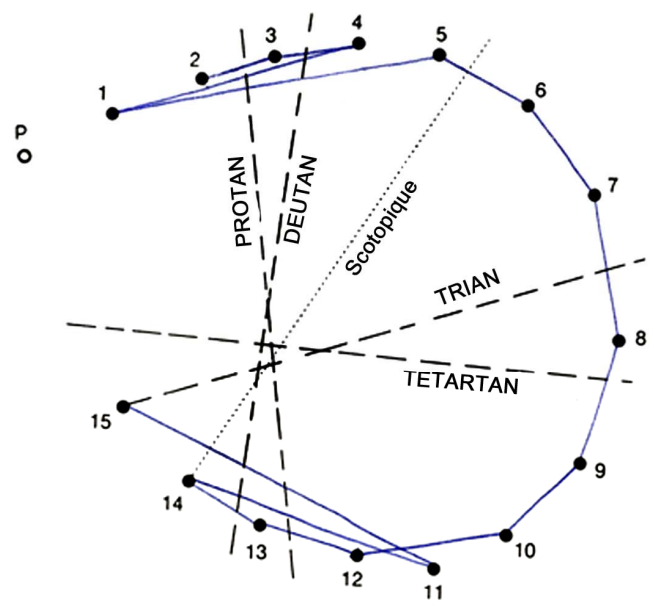

(b)

Figure 2. (a) Fransworth-Munsell D-15 Hue test on right eye indicating a very mild atypical color deficiency; (b) Fransworth-Munsell D-15 Hue test on left eye also indicating a very mild atypical color deficiency.

Few days following patient's hospitalization his best corrected visual acuity decreased progressively (OD and OS 3/10) and he paroxysmally experienced illusory left homonymous transpositions of subjects viewed in the right homonymous visual field. The transposition occurred from normal to the defective visual field. This condition is called visual allesthesia. Patient did not describe palinopsia (i.e., persistence or recurrence of visual images after the exciting stimulus object was removed) of the illusory images on the homonymous left half-field but he described spatial repetition of these illusory images on the homonymous left half-field (one image behind and sideling the other).

\section{Discussion}

The initial symptom experienced by the patient was color perception disturbance. Although patient's visual field evaluation by automated perimetry-effectuated one day after his hospitalization—revealed homonymous left hemianopsia, patient did not refer any visual field defect. In the following days, during his hospitalization, his visual acuity was decreased and he experienced visual allesthesia paroxysmally.

Visual allesthesia is a rare condition experienced by patients with temporal or occipital cortex damage. In visual allesthesia (Greek allache $=$ elsewhere and aisthesis = perception) visual images are transposed from one homonymous half-field of visual field to another. Occasionaly the visual symptoms may be transposed from the lower to upper quadrant, or viceversa. This rare phenomenon is often accompanied by palinopsia. Visual allesthesia, along with palinopsia, differs from epileptic visual illusions and hallucinations from occipital and temporal lesions [5].

Some authors have reported these rare phenomena of visual allesthesia and palinopsia, but pathophysiology still remains obscure. Visual allesthesia is reported in a patient with right falcotentorial meningioma [6]. Authors refer that administration of anticonvulsant medications resulted in remission of visual allesthesia [6,7] and after surgical resection of the tumor visual allesthesia was completely disappeared. In this case [6], tumor-induced 

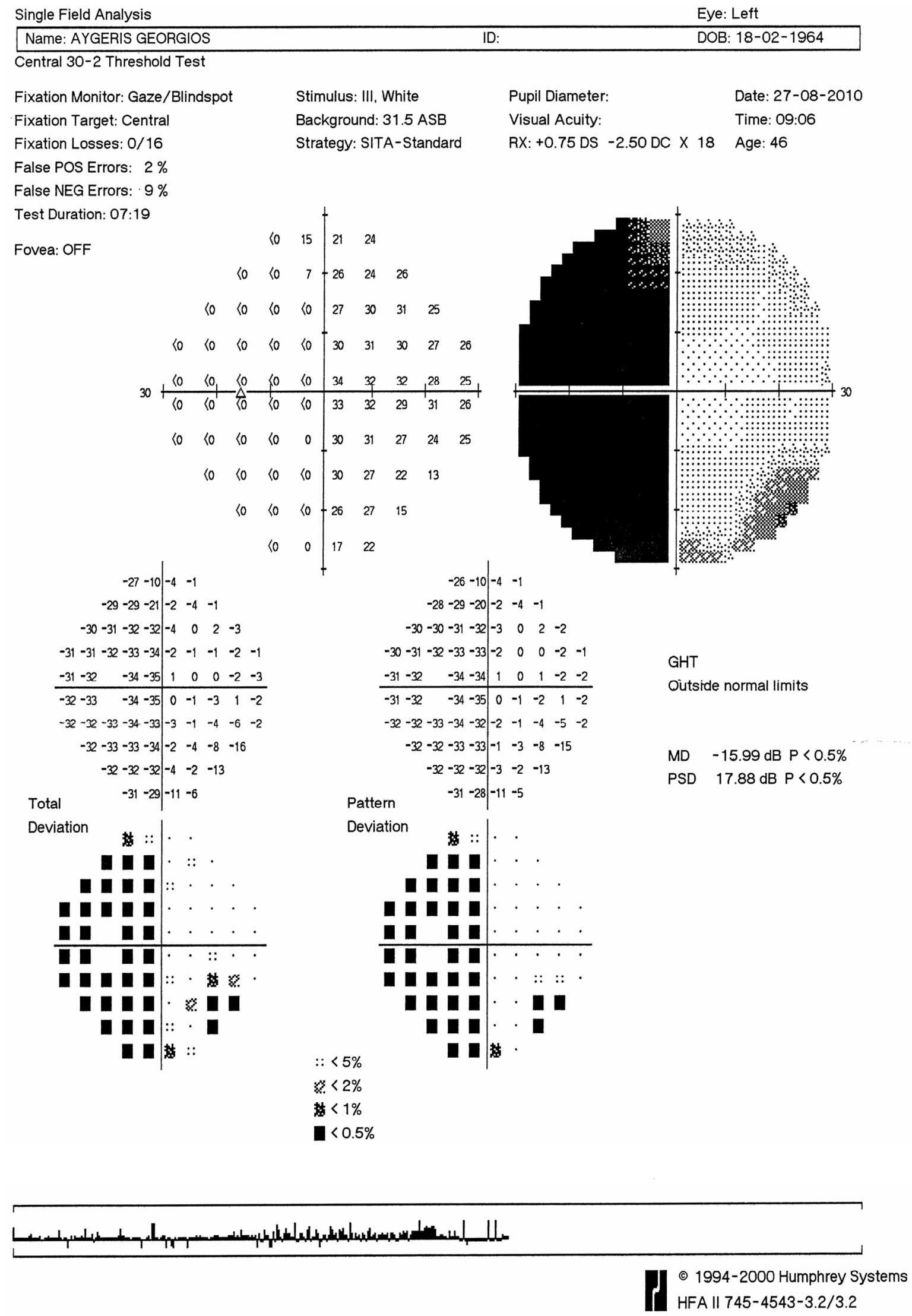

Figure 3. Humphrey visual field analysis, program 30-2. Homonymous left hemianopsia. 


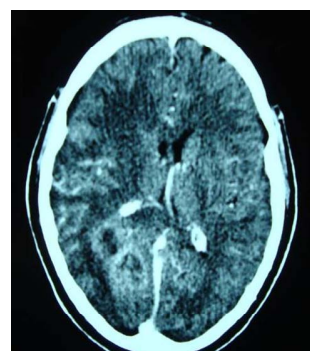

Figure 4. Axial CT-scan with intravenous contrast. This image reveals a mass (glioma) located inside the right temporal-occipital lobe with peripheral zone enhancement and central necrosis, mild compressive effects on the ventricular system without midline shift.

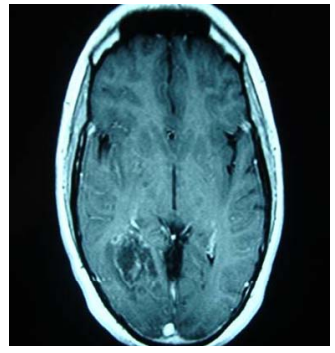

(a)

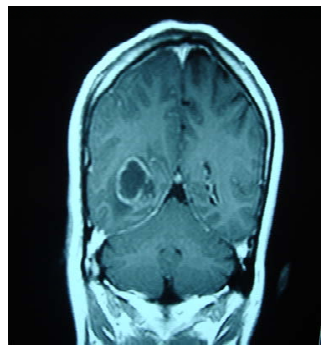

(b)
Figure 5. MRI with intravenous contrast. (a) T1-weighted transversal MRI with intravenous contrast. This image reveals a mass (GBM) located inside the occipital lobe with 3 $\times 6 \mathrm{~cm}$ of dimension, which is surrounded by edema with compressive effects on the ventricular system; (b) T1weighted coronal MRI with intravenous contrast. This image reveals heterogeneous and ring enhancement. The central hypodense core represents necrosis, the contrast-enhancing ring is composed of highly dense neoplastic cells with abnormal vessels permeable to contrast agents. The peripheral zone of non enhancing low attenuation is vasogenic edema containing varying numbers of invasive tumor cells.

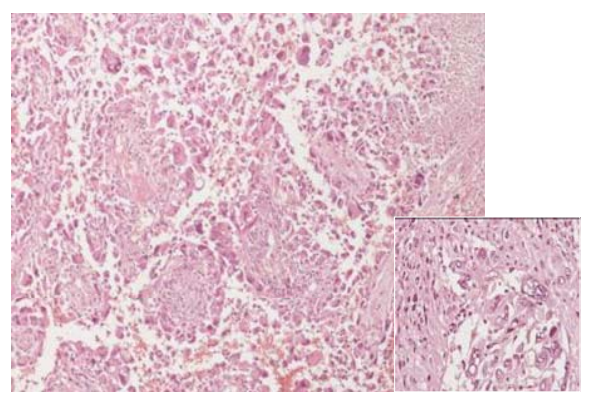

Figure 6. Histopathologic slide demonstrating patient's GBM. Lesion is composed of giant pleomorphic astrocytic cells. Necrosis and endothelial proliferation is observed. Immunohistochemically neoplastic astrocytes are positive for glial fibrillary acidic protein (GFAP) and CD 34. Using epidermal growth factor receptor (EGFR) staining diffuse cytoplasmatic and less clear membrane positivity is observed. Using p53-protein (tumor suppressor protein) staining focal nuclear positive staining is observed in some cells. Cellular proliferation index ki-67 approximate $20 \%$ (hot spot). compression effects on visual cortex area Brodmann 8 and 9 are considered as causative of visual allesthesia. Another case of visual allesthesia is reported in a patient with right temporo-occipital arteriovenous malformation [8]. In contrast, in this case, visual allesthesia was presented 6 months after surgery. Occipital calcified cysticercu, right occipital infarct, right parieto-occipital arteriovenous malformation [6], and epilepsia partialis continua $[9,10]$ are also reported in causing visual allesthesia. Pathophysiology of visual allesthesia still remains unknown. Visual allesthesia usually results from right hemisphere lesions with left-sided neglect or extinction [9, 11,12]. Visual allesthesia may result from interhemispheric transfer of vision from normal to a partially defected left visual field [7,13,14], in association with sustained neural activity in the controlateral parietal cortex [13]. Hallucinations from temporal and occipital lesions differ from visual allesthesia which implicates a parietal localization [5].

We also underline that in the present case report-patient, color perception disturbance took place prior to visual acuity decrease, the patient revealed the experience of visual allesthesia a few days after hospitalization and also he did not notice left homonymous hemianopsia which was revealed by standard automated perimetry.

\section{REFERENCES}

[1] P. Kleihues, P. C. Burger and W. K. Cavenee, "Glioblastoma,” In: P. Kleihues and W. K. Cavenee, Eds., Pathology and Genetics of Tumors of the Nervous System, Lyon, 1997, pp. 16-24.

[2] J. N. Rich, C. Hans, B. Jones, et al., "Gene Expression Profiling and Genetic Markers in Glioblastoma Survival,” Cancer Research, Vol. 65, No. 10, 2005, pp. 4051-4058.

[3] H. Ohgaki and P. Kleihues, "Population-Based Studies on Incidence, Survival Rates, and Genetic Alterations in Astrocytic and Oligodendroglial Gliomas," Journal of Neuropathology \& Experimental Neurology, Vol. 64, No. 6 2005, pp. 479-489.

[4] C. J. Farrell and S. R. Plotkin, "Genetic Causes of Brain Tumors: Neurofibromatosis, Tuberous Sclerosis, von Hippel-Lindau, and Other Syndromes,” Neurologic Clinics, Vol. 25, No. 4, 2007, pp. 925-946 doi:10.1016/j.ncl.2007.07.008

[5] C. G. Bien, F. O. Benninger, H. Urbach, J. Schramm, M. Kurthen and C. E. Elger, "Localizing Value of Epileptic Visual Auras,” Brain, Vol. 123, No. 2, 2000, pp. 244-253. doi:10.1093/brain/123.2.244

[6] T. Arai, K. Irie, M. Akiyama, T. Kamikubo, M. Nakajima, H. Sakai and T. Abe, "A Case of Falcotentorial Meningioma with Visual Allesthesia,” No To Shinkei, Vol. 54, No. 3, 2002, pp. 255-259.

[7] L. Jacobs, "Visual Allesthesia,” Neurology, Vol. 30, No. 10, 1980, pp. 1059-1063. doi:10.1212/WNL.30.10.1059

[8] M. Nakajima, M. Yasue, N. Kaito, T. Kamikubo and H. 
Sakai, "A Case of Visual Allesthesia," No To Shinkei, Vol. 43, No. 11, 1991, pp. 1081-1085.

[9] J. Mendez, "Palinopsia and Visual Allesthesia," International Journal of Neuroscience, Vol. 32, No. 3-4, 1987, pp. 775-782. doi:10.3109/00207458709043332

[10] M. F. Mendez and J. W. Chen, "Epilepsy Partialis Continua with Visual Allesthesia,” Journal of Neurology, Vol. 256, No. 6, 2009, pp. 1009-1011.

[11] M. B. Bender, M. F. Shapiro and H. L. Teuber, "Allesthesia and Disturbance of the Body Scheme," Archives of Neurology and Psychiatry, Vol. 62, No. 2, 1949, pp. 222235.
[12] K. J. Meador, M. E. Allen, R. J. Adams and D. W. Loring, "Allochiria vs. Allesthesia. Is There a Misperception?" Archives of Neurology, Vol. 48, 1991, pp.546-549. doi:10.1001/archneur.1991.00530170110029

[13] A. Shewmon, "Visual Allesthesia,” Neurology, Vol. 31, No. 4, 1981, p. 496.

[14] K. J. Burcham, J. V. Corwin, M. L. Stoll and R. L. Reep, "Disconnection of Medial Agranular and Posterior Parietal Cortex Produces Multimodal Neglect in Rats,” Behavioural Brain Research, Vol. 86, No. 1, 1997, pp. 41-47. doi:10.1016/S0166-4328(96)02241-3 